\title{
Dinâmica populacional da mariposa oriental em pomares de pessegueiro e ameixeira(1)
}

\author{
Eduardo Rodrigues Hickel(2), Gabriel Rodrigues Hickel(3), Og Francisco Fonseca de Souza( ${ }^{(4)}$,
} Evaldo Ferreira Vilela(4) e Octavio Miramontes ${ }^{(5)}$

\begin{abstract}
Resumo - O objetivo deste trabalho foi estudar a dinâmica populacional da mariposa oriental [Grapholita molesta (Busk)] em pomares de pessegueiro e ameixeira. Os adultos foram monitorados em dois pomares de pessegueiro e um de ameixeira por cinco anos. Séries temporais equivalentes de temperaturas máximas e mínimas foram correlacionadas aos dados de flutuação populacional para se aferir a correspondência entre a ocorrência da praga e as flutuações de temperatura. A análise de Fourier das séries temporais das armadilhas de coleta de mariposas revelou dinâmicas cíclicas, com período principal médio de 53,91 semanas. Um período menor médio, de 7,45 semanas, também ficou evidente e se aproxima do tempo médio de geração do inseto. A análise do espectro de potência das séries temporais, com e sem periodicidade, revelou que a dinâmica populacional de G. molesta possui um componente regular, caracterizado pelos períodos observados, e outro componente irregular, reminiscente de ruído $1 /$ f, caracterizado pelas variações de amplitude, aparentemente irregulares, na ocorrência do inseto. A combinação desses dois componentes ocasiona um processo com domínio de baixas freqüências, próprio das dinâmicas periódicas ruidosas. Os ciclos de aparecimento da praga ocorrem em fase com os ciclos de flutuação de temperaturas máximas e mínimas.
\end{abstract}

Termos para indexação: Grapholita molesta, Prunus, população de inseto, inseto nocivo, praga das plantas.

\section{Population dynamics of oriental fruit moth in peach and plum orchards}

Abstract - The objective of this work was to study the populational dynamics of oriental fruit moth [Grapholita molesta (Busk)] in peach and plum orchards. Adults were monitored in two peach and one plum orchards for five years. Equivalent time series of maximum and minimum temperatures were correlated to the population fluctuation data to verify the correspondence between pest occurrence and temperature fluctuations. A Fourier analysis of the moth time series revealed cyclic dynamics, with a main average period of 53.91 weeks. Another mean short period of 7.45 weeks was also obtained, and approximates to the mean pest generation time. The power spectrum analysis of the time series, with and without the main periodicity, revealed that G. molesta population dynamics has one regular component characterized by the observed periods, and one irregular component, reminiscent of $1 / \mathrm{f}$ noise, characterized by the observed apparently irregular amplitude oscillations in the insect occurrence. The combination of components generate a process in the low frequency domain, consistent with noisy periodic dynamic. The pest cycles occurred in phase with the maximum and minimum temperature fluctuations

Index terms: Grapholita molesta, Prunus, insect population, pest insects, pests of plants.

(1) Aceito para publicação em 26 de novembro de 2002.

${ }^{(2)}$ Universidade Federal de Viçosa (UFV), Dep. de Biologia Animal, CEP 36570-000 Viçosa, MG. Bolsista do CNPq. E-mail: hickel@epagri.rct-sc.br

(3) Instituto Nacional de Pesquisas Espaciais, Div. de Astrofísica, Caixa Postal 515, CEP 12202-970 São José dos Campos, SP Bolsista da Fapesp. E-mail: hickel@das.inpe.br

(4)UFV. E-mail: og.souza@ufv.br, evilela@ufv.br

(5) Universidad Nacional Autónoma de México, Instituto de Física, Ciudad Universitaria, México 01000 DF. E-mail: octavio@fisica.unam.mx

\section{Introdução}

A mariposa oriental [Grapholita molesta (Busk, 1916)] (Lepidoptera: Tortricidae), também conhecida como grafolita ou broca-dos-ponteiros, é uma das pragas que mais prejudicam o cultivo do pessegueiro e da ameixeira no Brasil (Salles, 1984). Também incide sobre outras frutíferas, principalmente da família Rosaceae, como macieira, marmeleiro, pereira e nespereira. Amplamente disseminada por todas 
as regiões produtoras de frutas de clima temperado, esta praga causa perdas expressivas, quer na fase da colheita quanto na implantação dos pomares, quando afeta o crescimento normal das plantas.

A elucidação do feromônio sexual da espécie, que intermedeia a atração entre sexos, permitiu o desenvolvimento da técnica de monitoramento de adultos com armadilhas, e assim a realização de vários estudos de flutuação populacional (Phillips, 1973; Forno et al., 1975; Silveira Neto et al., 1981). Entretanto, uma das questões não elucidadas é a periodicidade de ocorrência de G. molesta nos pomares ao longo dos anos, importante no estabelecimento de ajustes e modelos matemáticos para previsão de eventos relacionados à flutuação populacional do inseto. Outra questão a elucidar, é em relação à natureza das oscilações na coleta de indivíduos. Embora o comportamento caótico seja potencialmente possível em dinâmicas populacionais (Logan \& Allen, 1992; Constantino et al., 1995; Rai \& Schaffer, 2001), alguns estudos revelam que essas dinâmicas estão no limiar entre ordem e desordem, em que as oscilações ocorrem com escalamento 1/f (a potência da oscilação é proporcional ao inverso da freqüência), com domínio de baixas freqüências (Halley, 1996; Miramontes \& Rohani, 1998; Solé et al., 1999; Skokov et al., 2000).

O objetivo deste trabalho foi estudar a dinâmica populacional da mariposa oriental em pomares de pessegueiro e ameixeira.

\section{Material e Métodos}

Dados de coleta semanal em dois pomares de pessegueiro e um de ameixeira, de adultos de G. molesta em armadilhas de feromônio, por período de cinco safras agrícolas (outubro de 1994 a janeiro de 1999), foram utilizados nas análises. Os pomares eram da coleção de cultivares da Estação Experimental de Videira, em Videira, SC. O primeiro pomar de pessegueiro (pêssego 1) continha plantas de segunda brota no início do monitoramento e o segundo pomar de pessegueiro (pêssego 2) e o de ameixeira (ameixa) continham plantas em produção. Os tratos culturais e fitossanitários foram executados conforme Epagri $(1995,1996)$, com aplicação de inseticida no período de monitoramento, principalmente para controle da mosca-das-frutas Anastrepha fraterculus (Wied.)
A técnica de monitoramento de G. molesta empregada consistiu na instalação de armadilhas de feromônio tipo Delta, com $1 \mathrm{dm}^{2}$ de área de coleta, na proporção de uma armadilha por hectare de pomar (Rice et al., 1982; Salles, 1984). As armadilhas foram suspensas a $1,60 \mathrm{~m}$ de altura no interior da copa das árvores e em seu interior foram instalados um dispositivo dispersor de feromônio sexual sintético e, dependendo da montagem da armadilha, um cartão adesivo. Os dispersores de feromônio foram trocados a cada sete semanas, em média, e os cartões adesivos ou toda a armadilha quando houve acúmulo de detritos ou deterioração (Hickel \& Ducroquet, 1998).

As armadilhas foram instaladas nos pomares na primeira semana de agosto, com exceção da safra 1994/1995, a fim de detectar a primeira emergência de adultos originários das lagartas em diapausa (Rice et al., 1982; Hickel \& Ducroquet, 1998), e permaneceram no campo até a última semana de julho do ano seguinte, com exceção da safra 1998/1999. A inspeção das armadilhas foi feita uma vez por semana, quando foram registradas e retiradas do cartão adesivo as mariposas capturadas.

Para verificar a periodicidade de ocorrência de G. molesta, os valores de coleta de mariposas foram organizados em planilha eletrônica e transferidos para o software DFT, que procede a análise transformada discreta de Fourier (DFT), ou seja, transforma os dados do domínio temporal para o domínio das frequiências. Os valores de período $(\mathrm{P})$, tempo para ocorrência do primeiro máximo $\left(\mathrm{T}_{\max }\right)$, amplitude média $(\mathrm{R})$ e a média das contagens $\left(\mathrm{a}_{0}\right)$, foram calculados para o melhor ajuste senoidal às variações da ocorrência do inseto pela seguinte equação:

$F(x)=a_{0}+R x \cos \left(2 \Pi x \frac{\left(x-T_{\max }\right)}{P}\right)$

A estimativa da ocorrência dos máximos populacionais $\left(\mathrm{T}_{\mathrm{i}}\right)$ foi obtida pela equação 2 utilizando-se o período $(\mathrm{P})$ e o tempo para ocorrência do primeiro máximo de contagem $\left(\mathrm{T}_{\max }\right)$. Essas estimativas foram confrontadas com os picos observados na flutuação populacional, obtendo-se a significância pelo teste do $\chi^{2}$

$\mathrm{T}_{\mathrm{i}}=(\mathrm{ix} \mathrm{P})+\mathrm{T}_{\max }$,

em que: $\mathrm{i}=0,1,2, \ldots, \mathrm{n}$.

Os valores das probabilidades relativas de cada freqüência nos espectros de potência das séries temporais, com e sem as periodicidades observadas, para analisar o comportamento das oscilações de amplitude (ruído) nos períodos de ocorrência de G. molesta, foram obtidos por meio do software DFT. Esses valores foram plotados em escala log-log, obtendo-se em seguida o ajuste linear pelo método dos mínimos quadrados. O coeficiente angular da 
equação linear $(\alpha)$ foi utilizado para classificar o comportamento da flutuação populacional (Solé et al., 1999; Clark, 2001). Na formação das séries temporais sem as periodicidades observadas, para verificar a natureza do ruído permeado nas séries, o período principal foi subtraído diretamente dos respectivos periodogramas obtidos no DFT, gerando-se assim as séries residuais.

As séries temporais de captura de mariposas foram analisadas também com o software CDA, que realiza o teste IFS (Iterated Function System) e calcula o expoente de Hurst, parâmetros utilizados para confirmar a natureza das oscilações na coleta de indivíduos (Miramontes \& Rohani, 1998)

Séries temporais equivalentes de temperaturas máximas e mínimas, obtidas na Estação Experimental de Videira, foram também processadas pelo DFT e CDA, para se aferir a existência das mesmas dinâmicas nesta variável ambiental. Essas séries foram também analisadas com o software DCF para análise de correlação/autocorrelação, a fim de verificar a correspondência entre a ocorrência da praga e as flutuações de temperatura (Turchin et al., 1991).

\section{Resultados e Discussão}

Houve intensa variação no número de mariposas capturadas em cada ciclo de ocorrência e semanas com número elevado de mariposas capturadas se intercalam com semanas com baixas capturas de indivíduos, aparentemente denotando os ciclos de geração (Knell, 1998) (Figura 1). Períodos longos com ausência de capturas correspondem às épocas de frio hibernal.

A análise DFT revelou o período aproximado de um ano para a ocorrência de G. molesta nos pomares monitorados, embora outras periodicidades sem maior significado biológico também tenham sido verificadas (Tabela 1). Apenas os menores períodos significativos gerados na DFT é que se aproximam do ciclo de geração do inseto, estimado entre 5,7 a 8 semanas a $20^{\circ} \mathrm{C}$ (Grelmann, 1991) e são importantes para caracterizar a intermitência na ocorrência de indivíduos. A estimativa dos máximos populacionais apresentou um desvio progressivo, de maior valor para os últimos máximos calculados (Tabela 2).

$\mathrm{O}$ ajuste linear do espectro de potência das séries temporais na íntegra resultou em coeficientes $\alpha$ próximos de -1 (Figura 2); valor este característico da distribuição de freqüências $1 /$ f. O mesmo ajuste, para as séries residuais, resultou em coeficientes $\alpha$ mais afastados do valor -1 , porém ainda com forte tendência para distribuição $1 / f$. O ruído $1 / \mathrm{f}$ permaneceu forte nos intervalos de tempo reduzidos (20 semanas ou menos), em que os eventos maiores parecem se equivaler em escala aos eventos menores. Tal fenômeno pode ser observado no intervalo entre 50 e 75 semanas, cujo pico principal aparenta ser uma projeção em escala dos picos anteriores (Figura 1).

Apesar de pouco aparente, pelo reduzido número de valores das séries temporais, é possível notar, no resultado do teste IFS, arranjos triangulares de pontos que se repetem em escalas ampliadas a partir de um dos cantos e pontos acumulados na diagonal do quadro (Figura 3). Esse arranjo de pontos é similar ao produzido por séries de oscilação 1/f (Miramontes \& Rohani, 1998). O coeficiente de Hurst (H), que fornece uma indicação da persistência na série temporal, ou seja, a ocorrência futura ou não de tendências passadas na série temporal, situou-se na faixa esperada para séries com ruído $1 / \mathrm{f}(0<\mathrm{H}<0,5)$. Em relação ao pomar pêssego 1 , série na íntegra e série descontada do período principal, os valores de $\mathrm{H}$ foram 0,16 e 0,28 , respectivamente; nos pomares pêssego 2 e ameixa, esses coeficientes foram iguais a 0,27 e 0,26 e 0,24 e 0,30 , respectivamente.

A análise de autocorrelação permitiu evidenciar os mesmos períodos anuais de ocorrência da praga, já verificados com o DFT, porém outros períodos bianuais, em torno de 104 semanas, também ficaram evidentes (Figura 4). Esses períodos bianuais provavelmente não ficam evidentes com Fourier, em razão dos harmônicos que se igualam a duas vezes o período principal. Esses ciclos bianuais surgem em razão de dois adiantamentos na ocorrência da praga, em cerca de 100 semanas e após 200 semanas, intercalados por anos de ocorrência normal (Figura 1) e seu significado pode ser tema para novas prospecções científicas.

Os registros de temperatura também apresentaram periodicidade anual $(\mathrm{P}=52,3$ semanas e $\mathrm{P}=51,88$ semanas para as séries de temperatura máxima e mínima, respectivamente), com os períodos de temperatura acima da média da série coincidindo com os períodos de ocorrência da praga (Figura 5). Não houve defasagem de tempo entre a variação na ocorrência de adultos e a variação na tempe- 

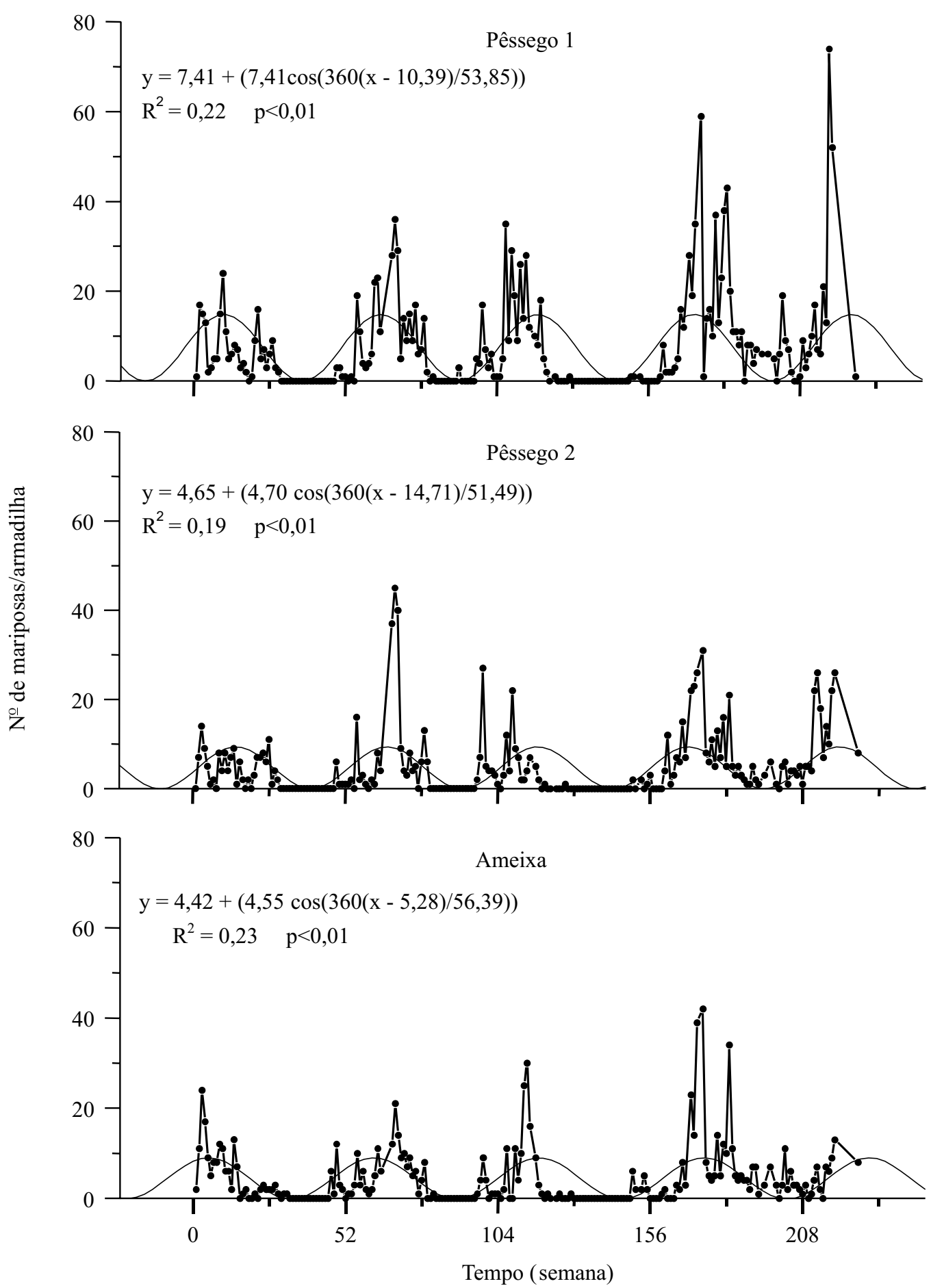

Figura 1. Flutuação populacional de Grapholita molesta em Videira, SC $(\bullet)$ e senóides geradas a partir dos períodos principais obtidos com o software DFT (linha cheia fina). 
Tabela 1. Períodos significativos $(\mathrm{p}<0,01)$ e parâmetros associados (média \pm desvio-padrão) resultantes da análise de Fourier das séries temporais de coleta de Grapholita molesta em armadilhas de feromônio em pomares de pêssego (1 e 2) e ameixa, em Videira, SC.

\begin{tabular}{|c|c|c|c|c|}
\hline $\begin{array}{l}\text { Sequiência de } \\
\text { períodos }\end{array}$ & $\begin{array}{l}\text { Período } \\
\text { (semana) }\end{array}$ & $\begin{array}{l}\text { Tempo do primeiro } \\
\text { máximo (semana) }\end{array}$ & $\begin{array}{l}\text { Amplitude média } \\
\text { (indivíduo) }\end{array}$ & $\begin{array}{l}\text { Média das capturas } \\
\text { (indivíduo) }\end{array}$ \\
\hline \multicolumn{5}{|c|}{ Pêssego 1} \\
\hline 1 & $99,82 \pm 5,43$ & $88,07 \pm 3,55$ & $4,66 \pm 1,04$ & $7,41 \pm 0,74$ \\
\hline $2^{(1)}$ & $53,85 \pm 0,92$ & $10,39 \pm 1,11$ & $7,41 \pm 0,96$ & $7,41 \pm 0,68$ \\
\hline 3 & $36,88 \pm 0,63$ & $32,56 \pm 1,12$ & $5,35 \pm 1,02$ & $7,43 \pm 0,72$ \\
\hline 4 & $21,25 \pm 0,38$ & $5,92 \pm 1,17$ & $3,07 \pm 1,06$ & $7,41 \pm 0,75$ \\
\hline $5^{(2)}$ & $7,44 \pm 0,05$ & $2,72 \pm 0,42$ & $3,02 \pm 1,07$ & $7,37 \pm 0,75$ \\
\hline \multicolumn{5}{|c|}{ Pêssego 2} \\
\hline 1 & $111,47 \pm 7,45$ & $81,45 \pm 4,36$ & $2,88 \pm 0,71$ & $4,80 \pm 0,50$ \\
\hline $2^{(1)}$ & $51,49 \pm 0,91$ & $14,71 \pm 1,16$ & $4,70 \pm 0,66$ & $4,65 \pm 0,47$ \\
\hline 3 & $37,01 \pm 0,63$ & $29,77 \pm 1,12$ & $3,64 \pm 0,69$ & $4,76 \pm 0,49$ \\
\hline 4 & $20,86 \pm 0,34$ & $6,44 \pm 1,06$ & $2,25 \pm 0,72$ & $4,74 \pm 0,51$ \\
\hline $5^{(2)}$ & $7,50 \pm 0,05$ & $2,24 \pm 0,50$ & $1,73 \pm 0,72$ & $4,73 \pm 0,51$ \\
\hline \multicolumn{5}{|c|}{ Ameixa } \\
\hline 1 & $90,23 \pm 4,34$ & $87,80 \pm 3,14$ & $2,83 \pm 0,62$ & $4,31 \pm 0,44$ \\
\hline $2^{(1)}$ & $56,39 \pm 0,97$ & $5,28 \pm 1,12$ & $4,55 \pm 0,56$ & $4,42 \pm 0,40$ \\
\hline 3 & $34,33 \pm 0,70$ & $6,00 \pm 1,33$ & $2,56 \pm 0,62$ & $4,18 \pm 0,44$ \\
\hline 4 & $20,78 \pm 0,31$ & $8,25 \pm 0,96$ & $2,18 \pm 0,63$ & $4,28 \pm 0,45$ \\
\hline $5^{(2)}$ & $7,41 \pm 0,05$ & $3,30 \pm 0,46$ & $1,64 \pm 0,64$ & $4,27 \pm 0,45$ \\
\hline
\end{tabular}

(1)Período mais provável ou principal. (2)Período que se ajusta aos ciclos de geração.

Tabela 2. Estimativa dos máximos populacionais (semanas) para a ocorrência anual de Grapholita molesta, em pomares de pêssego ( 1 e 2) e ameixa, a partir dos parâmetros do período principal resultante da análise de Fourier.

\begin{tabular}{|c|c|c|c|c|c|c|c|}
\hline \multirow{2}{*}{$\begin{array}{l}\text { Máximos } \\
\text { populacionais }\end{array}$} & \multicolumn{5}{|c|}{ Seqüência de máximos } & \multirow[t]{2}{*}{$\chi^{2}$} & \multirow[t]{2}{*}{$\mathrm{p}$} \\
\hline & 0 & 1 & 2 & 3 & 4 & & \\
\hline \multicolumn{8}{|c|}{ Pêssego 1} \\
\hline Estimado & $10,39 \pm 1,11$ & $64,24 \pm 2,03$ & $118,09 \pm 2,95$ & $171,94 \pm 3,87$ & $225,79 \pm 4,79$ & 1,70 & 0,79 \\
\hline Ocorrido & 10 & 69 & 107 & 174 & 218 & & \\
\hline Desvio & $-0,39$ & 4,76 & $-11,09$ & 2,06 & $-7,79$ & & \\
\hline \multicolumn{8}{|c|}{ Pêssego 2} \\
\hline Estimado & $14,71 \pm 1,16$ & $66,20 \pm 2,07$ & $117,69 \pm 2,98$ & $169,18 \pm 3,89$ & $220,67 \pm 4,80$ & 12,56 & 0,01 \\
\hline Ocorrido & 3 & 69 & 99 & 174 & 219 & & \\
\hline Desvio & $-11,71$ & 2,80 & $-18,69$ & 4,82 & $-1,67$ & & \\
\hline \multicolumn{8}{|c|}{ Ameixa } \\
\hline Estimado & $5,28 \pm 1,12$ & $61,67 \pm 2,09$ & $118,06 \pm 3,06$ & $174,45 \pm 4,03$ & $230,84 \pm 5,00$ & 2,60 & 0,63 \\
\hline Ocorrido & 3 & 69 & 114 & 174 & 219 & & \\
\hline Desvio & $-2,28$ & 7,33 & $-4,06$ & $-0,45$ & $-11,84$ & & \\
\hline
\end{tabular}



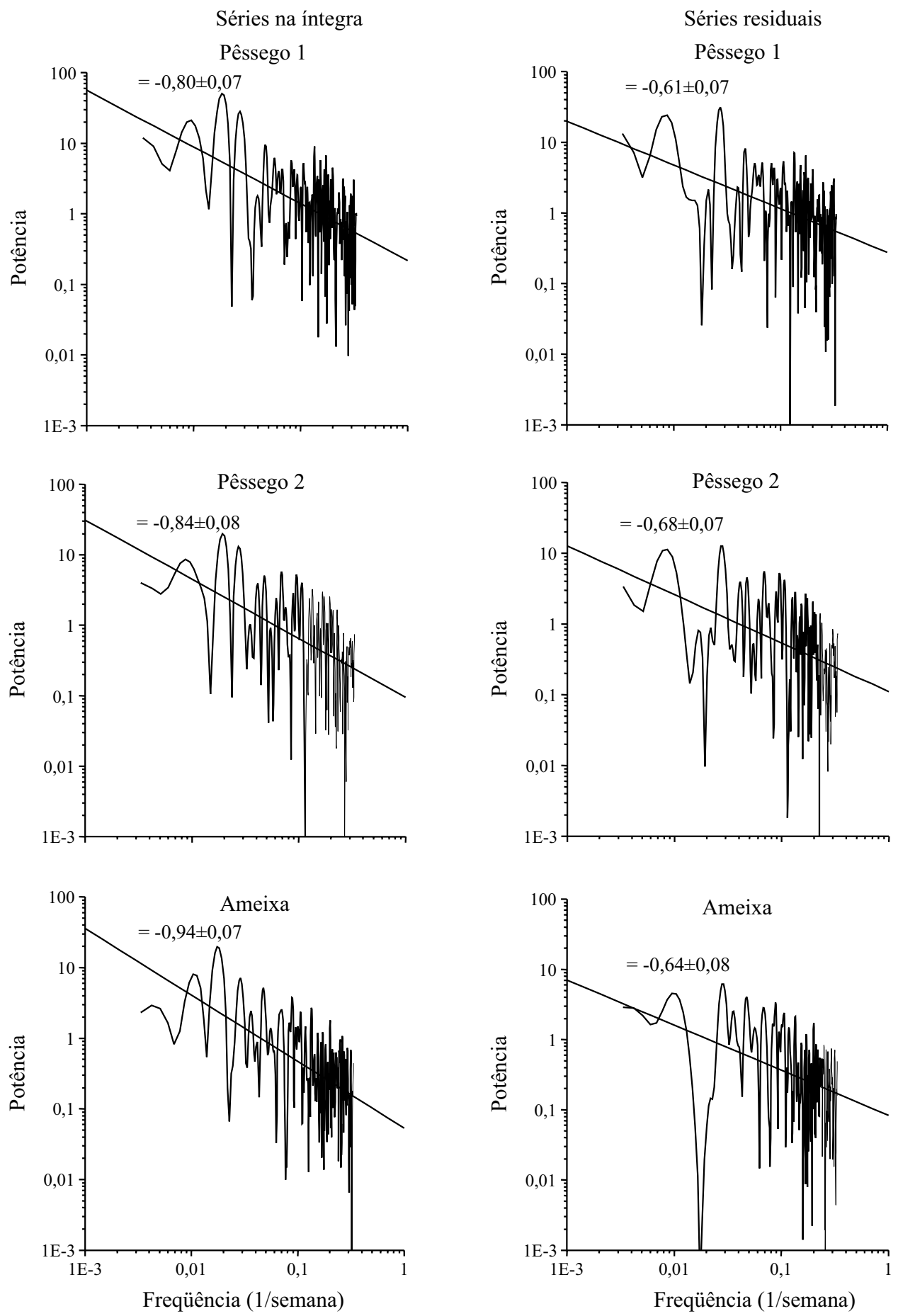

Figura 2. Espectros de potência das séries temporais de coleta de Grapholita molesta em Videira, SC, e respectivos ajustes lineares ( $\alpha$ : coeficiente angular da reta \pm desvio-padrão). 
IFS das séries na íntegra

Pêssego 1

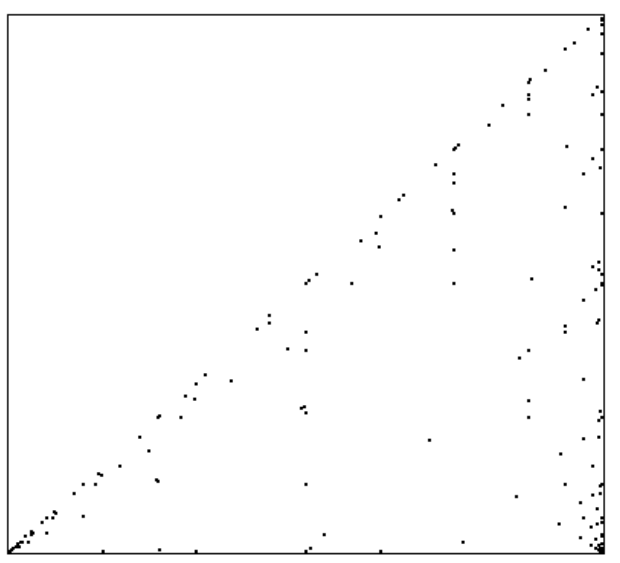

Pêssego 2

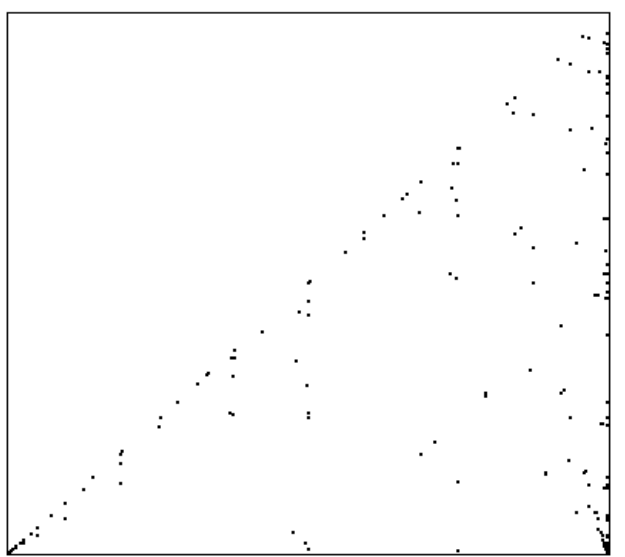

Ameixa

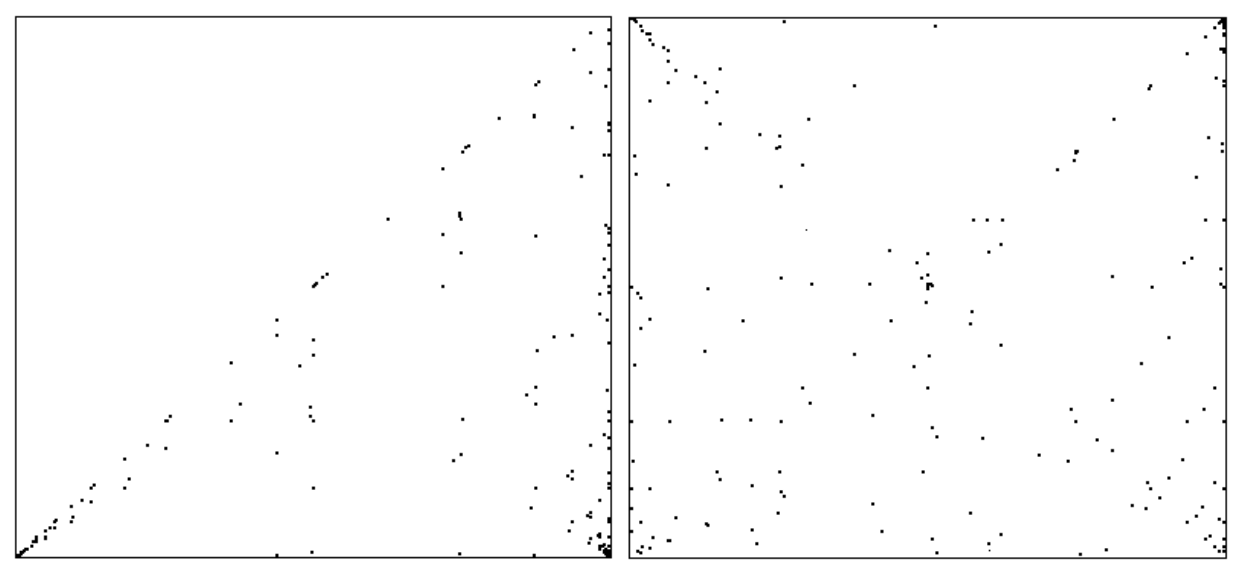

Figura 3. Quadros resultantes do teste iterativo da função de sistema (IFS), realizado pelo software CDA, aplicado às séries temporais de coleta de Grapholita molesta, em Videira, SC
IFS das séries residuais

Pêssego 1

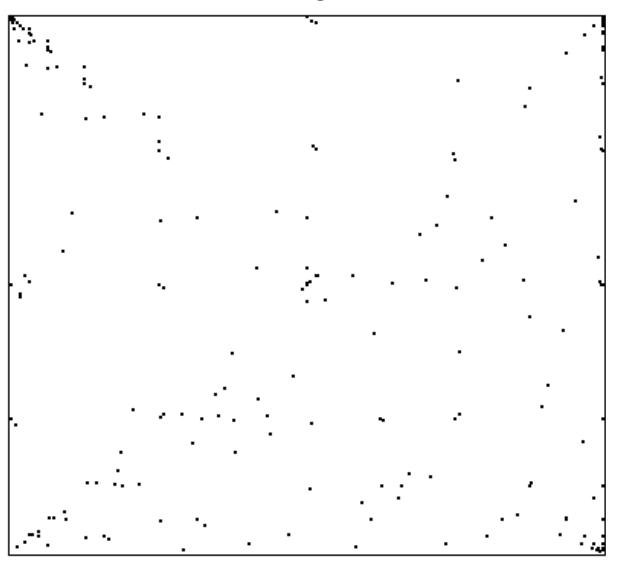

Pêssego 2

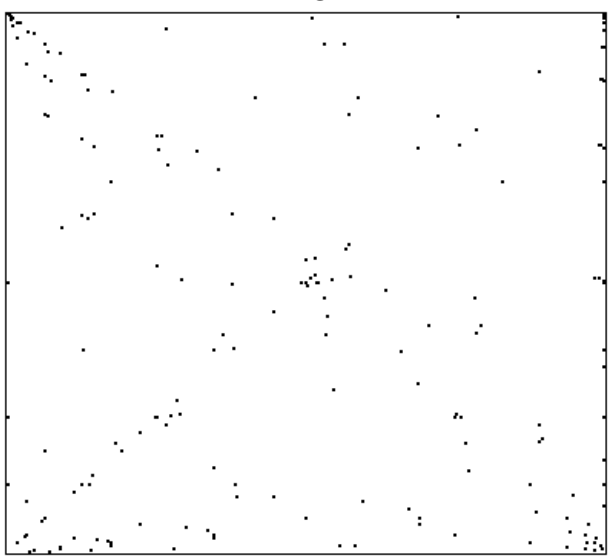

Ameixa

Pesq. agropec. bras., Brasília, v. 38, n. 3, p. 325-337, mar. 2003 

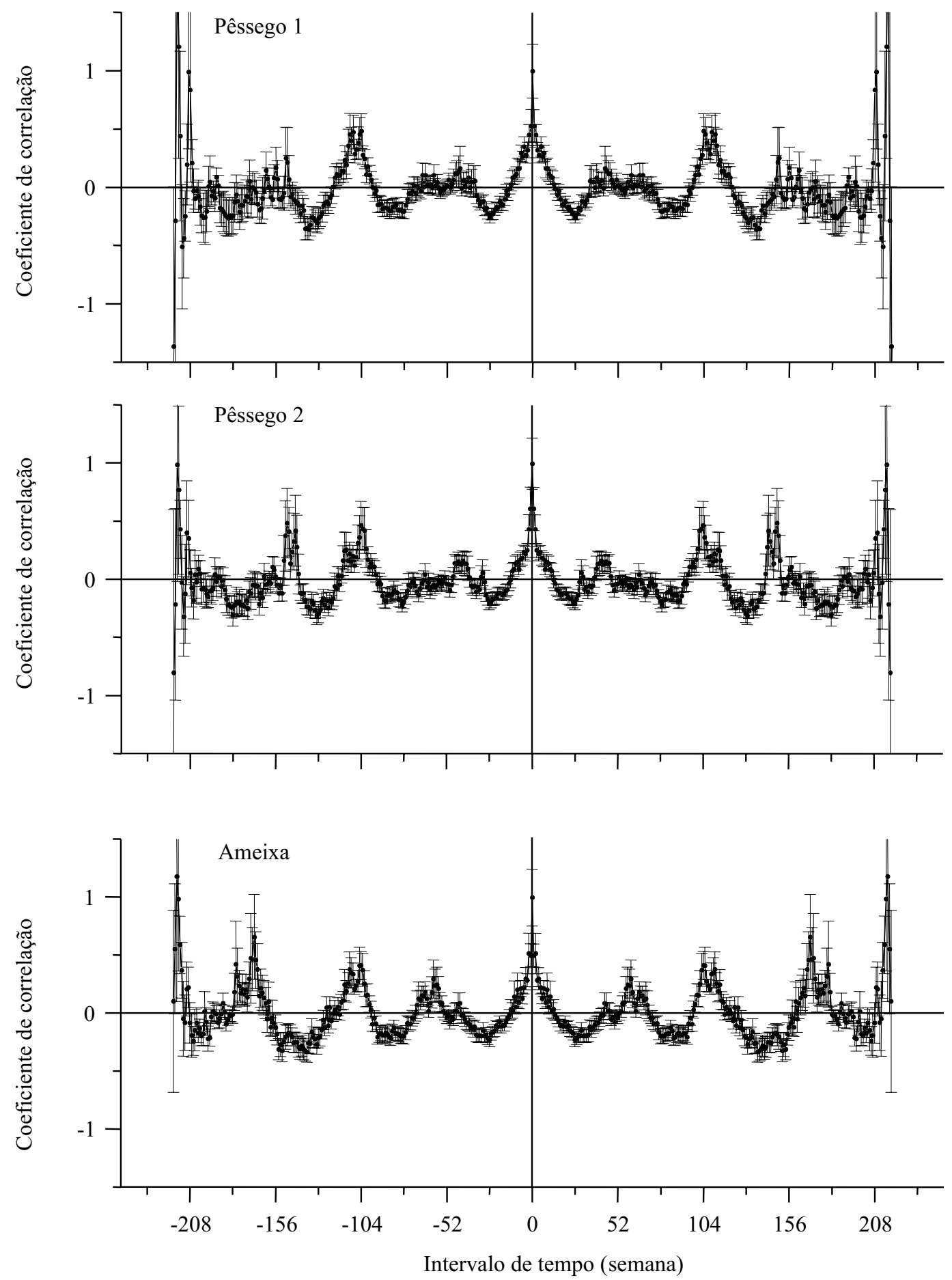

Figura 4. Gráficos de autocorrelação discreta das séries temporais de coleta de Grapholita molesta, em Videira, SC. 


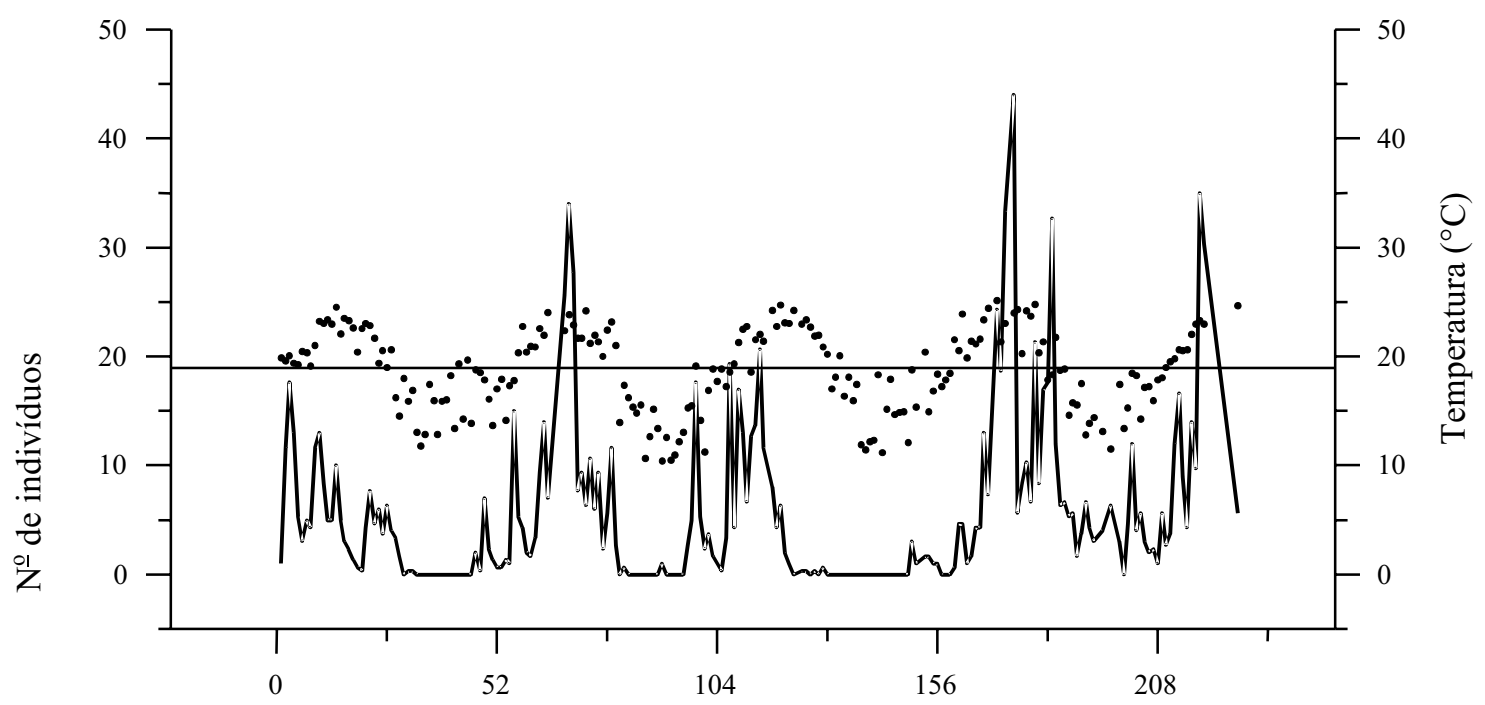

Tempo (semana)

Figura 5. Flutuação populacional média de Grapholita molesta (linha cheia) e flutuação da temperatura média semanal $(\bullet)$, em Videira, SC. A linha reta aos $18,9^{\circ} \mathrm{C}$ equivale à temperatura média da série temporal.

ratura, revelando que ambos os eventos ocorrem simultaneamente (Figura 6), ou seja, não necessariamente deve haver elevação prévia da temperatura para que haja ocorrência de mariposas. As oscilações de amplitude nos registros de temperatura também tendem para distribuição 1/f, com forte periodicidade ( $\alpha=-0,65 \pm 0,06 \mathrm{e} \alpha=-0,67 \pm 0,06$ para as séries de temperatura máxima e mínima, respectivamente).

Em seu ciclo anual, G. molesta sobrevive ao inverno em diapausa na fase larval, sob as cascas das árvores, em folhas aderidas aos ramos, em frutos mumificados ou sobre o solo, entre as folhas secas. Com o aumento da temperatura no final do inverno, as lagartas empupam e após 10 a 20 dias emergem os adultos (Salles, 1984). Dada a existência de diapausa durante o ciclo anual, é peculiar na flutuação populacional de G. molesta a ocorrência de levas de adultos (vôos), que vão se sobrepondo em gerações sucessivas durante a primavera e o verão. Assim, nos estudos de monitoramento, são característicos períodos de intensa coleta de mariposas, intercalados com períodos de baixa ou nenhuma coleta, sendo esta alternância de coletas uma característica importante para definir o controle da praga por meio do monitoramento (Rice et al., 1982).
As alternâncias de coletas num mesmo período de ocorrência do inseto podem ser atribuídas aos ciclos de geração (Knell, 1998; Azerefegne et al., 2001), os quais são muito evidentes em insetos que ocorrem em regiões de clima temperado, por causa do sincronismo de estágios de desenvolvimento após a diapausa, porém podem também ser evidenciados em espécies de clima tropical (Rice et al., 1982; Knell, 1998; Azerefegne et al., 2001). Tanto a presença de diapausa quanto de ciclos de geração ficaram evidentes no estudo de flutuação populacional de G. molesta, acarretando a ocorrência periódica e intermitente de adultos (Figura 1).

As oscilações no número de indivíduos, amostrados em estudos de flutuação populacional, normalmente são atribuídas a fatores relativos ao clima, principalmente temperatura (Turchin et al., 1991; Logan \& Allen, 1992; Berryman, 1996; Azerefgne et al., 2001). No entanto, diversos estudos de modelagem têm revelado a ocorrência de interações ecológicas, dinâmicas não lineares intrínsecas ou mesmo outros fatores não climáticos, regulando as oscilações de indivíduos (Turchin et al., 1991; Berryman, 1996; Grover et al., 2000; Rai \& Schaffer, 2001). Os surtos de ocorrência de 

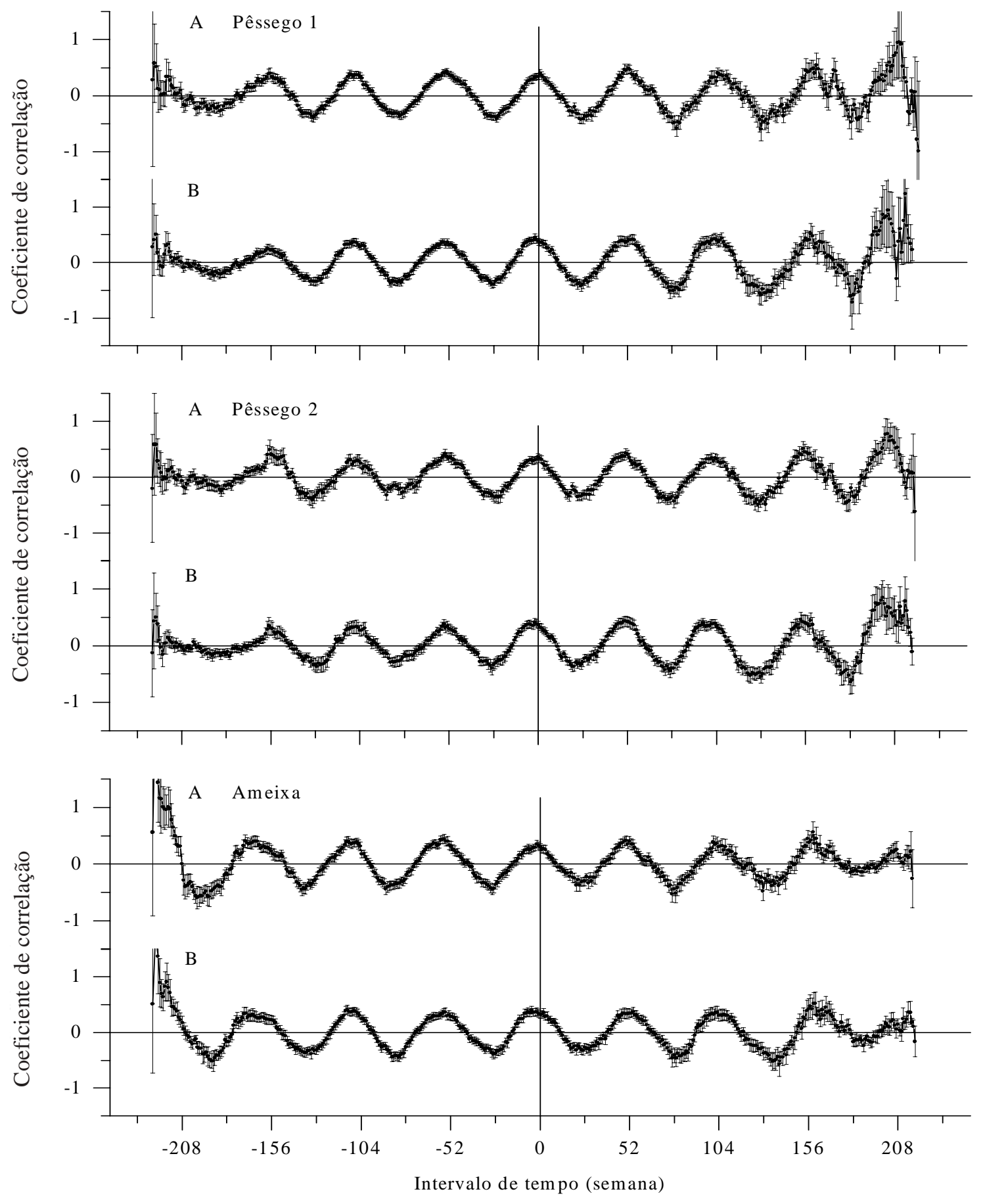

Figura 6. Correlação discreta das séries temporais de coleta de Grapholita molesta com as séries de temperatura máxima (A) e mínima (B) (médias semanais) obtidas em Videira, SC. 
G. molesta foram concomitantes com as flutuações de temperatura, e a ocorrência em fase dessas oscilações sugere que os registros de temperatura podem ser utilizados na obtenção de modelos ou ajustes para a previsão das épocas de ocorrência da praga. Apesar de ser notório que mais adultos da praga ocorrem quando está mais quente (Figura 7), as grandes oscilações da praga em curtos intervalos de tempo não têm correspondência nas séries de temperatura.

Estudos recentes têm demonstrado que o espectro da variação ambiental (se casual ou positivamente autocorrelacionado) não determina o espectro de variação das populações, que via de regra flutuam em baixa freqüência com alta amplitude. Esse padrão de flutuação é suficientemente forte para se manter ante as oscilações ambientais, embora possa ressonar quando as oscilações também ocorrem em baixa freqüência com alta amplitude (Constantino et al., 1998; Petchey, 2000).

As oscilações nas flutuações populacionais po- dem ser consideradas, por analogia, como um sinal, tal como ondas eletromagnéticas ou mecânicas, que sofrem uma série de interferências em seus respectivos percursos (Halley, 1996). Neste particular, atribuem-se cores para caracterizar os diferentes padrões. Assim, flutuações inteiramente aleatórias em freqüiencia e amplitude são ditas brancas (ou ruído branco), visto ser o branco a mistura de todas as cores. Outras possibilidades são o vermelho, cujas flutuações têm baixa freqüência com alta amplitude, e o azul, com alta freqüência e alta amplitude. A distribuição 1/f é tida como rosa, ou o vermelho desbotado, pelas interferências incorporadas.

No caso da flutuação populacional de insetos, as interferências podem ter origem nos efeitos de fatores ecológicos sobre a população (Turchin et al., 1991; Petchey, 2000), ampliados ou não por dinâmicas não-lineares intrínsecas, características dessas flutuações (Miramontes \& Rohani, 1998; Grover et al., 2000). Contudo, como sinal ou onda, existe uma fonte geradora cujo padrão de emissão se repe-

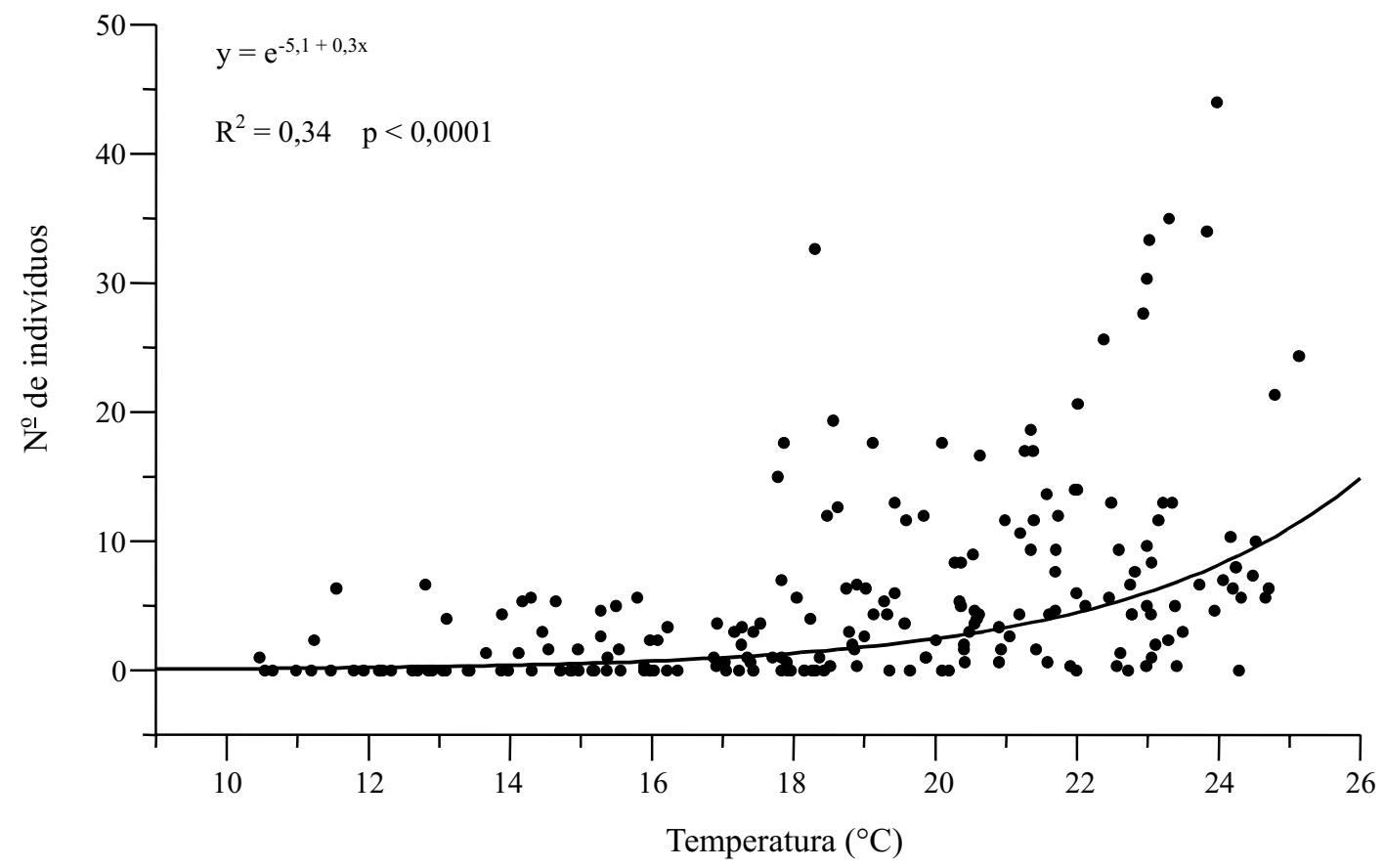

Figura 7. Regressão entre os valores médios de coleta de Grapholita molesta e de registro da temperatura média semanal em Videira, SC. 
te constantemente.

Mediante a análise do espectro de potência da série temporal, pode-se constatar que a dinâmica populacional de $G$. molesta possui uma componente regular, caracterizada nos períodos observados, e uma componente irregular, reminiscente de distribuição 1/f, caracterizada nas variações de amplitude na ocorrência do inseto. O teste IFS e o coeficiente de Hurst também corroboraram a detecção de ruído 1/f (perturbações com escalamento, imprevisíveis e independentes de densidade) permeado na série temporal de coleta de G. molesta.

A regularidade na dinâmica populacional de G. molesta advém do ciclo biológico do inseto e sua intermitência gerada pela diapausa hibernal; já o ruído $1 / f$ permeado pode estar sendo provocado pelas perturbações climáticas, ou então por dinâmicas nãolineares inerentes da flutuação populacional do inseto (processos demográficos). O processo amostral parece não provocar tais dinâmicas, pois séries temporais equivalentes, geradas por números aleatórios, anteriormente testadas pelos autores, apresentaram características de ruído branco.

Processos 1/f (com escalamento) são característicos de diversos fenômenos naturais, como abalos sísmicos e surtos de doenças, ou produzidos pela atividade humana, como a música ou flutuações no fechamento de bolsas de valores (Bak et al., 1988; Halley, 1996; Miramontes \& Rohani, 1998; Solé et al., 1999; Clark, 2001). Uma das implicações deste padrão com escalamento, para flutuações populacionais, é que um pico populacional não tende a ser precedido ou sucedido por outro pico populacional, devendo haver uma queda no número de indivíduos antes que picos sucessivos ocorram. Outra implicação é que grandes picos populacionais não se sucederão em curtos intervalos de tempo; assim, a ocorrência prolongada de um reduzido número de indivíduos passa a denotar que um grande pico populacional está prestes a ocorrer (Bak et al., 1988; Halley, 1996; Solé et al., 1999).

Tais informações podem ser fundamentais na estratégia de controle de G. molesta. Sabendo-se que picos populacionais serão sucedidos por decréscimo no número de indivíduos amostrados e que o máximo de posturas provavelmente ocorra nos picos populacionais, medidas de controle químico apli- cadas logo após os picos populacionais serão as mais prováveis de atingir eficientemente ovos e lagartas recém-eclodidas. Por sua vez, sendo uma dinâmica 1/ f, não haverá acúmulo de indivíduos na população com o passar do tempo, pois há pulsos de ocorrência de indivíduos, o que pode tornar inviável vincular a tomada de decisão do controle em níveis populacionais predefinidos. Essas conjecturas, entretanto, necessitam de mais estudos comprobatórios.

\section{Conclusões}

1. A mariposa oriental (Grapholita molesta) ocorre anualmente em pomares de pessegueiro e ameixeira na região do meio-oeste do Estado de Santa

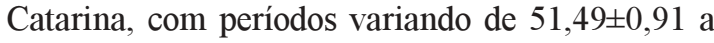
$56,39 \pm 0,97$ semanas

2. As oscilações na ocorrência de indivíduos seguem escalamento $1 / \mathrm{f}$ de ruído.

3. As flutuações populacionais de G. molesta ocorrem em fase com as flutuações de temperaturas máximas e mínimas.

\section{Agradecimentos}

Ao Dr. Francisco Jablonski (Inpe), por gentilmente ceder os softwares DFT e DCF utilizados neste estudo.

\section{Referências}

AZEREFEGNE, F.; SOLBRECK, C.; IVES, A. R. Environmental forcing and high amplitude fluctuations in the population dynamics of the tropical butterfly Acraea acerata (Lepidoptera:Nymphalidae). Journal of Animal Ecology, Oxford, v. 70, n. 6, p. 1032-1045, 2001.

BAK, P.; TANG, C.; WIESENFELD, K. Self-organized criticality. Physical Review A, Woodbury, v. 38, n. 1, p. 364-374, July 1988

BERRYMAN, A. A. What causes population cycles of forest Lepidoptera? Trends in Ecology and Evolution, Amsterdam, v. 11, n. 1, p. 28-32, Jan. 1996

CLARK, J. I. Fourier and power law analysis of structural complexity in cornea and lens. Micron, Amsterdam, v. 32, n. 3, p. 239-249, Apr. 2001 
CONSTANTINO, R. F.; CUSHING, J. M.; DENNIS, B.; DESHARNAIS, R. A. Experimentally induced transitions in the dynamic behavior of insect populations. Nature, London, v. 375, n. 6528, p. 227-230, May 1995.

CONSTANTINO, R. F.; CUSHING, J. M.; DENNIS, B.; DESHARNAIS, R. A.; HENSON, S. M. Resonant population cycles in temporally fluctuating habitats. Bulletin of Mathematical Biology, London, v. 60, n. 2, p. 247-273, Mar. 1998.

EPAGRI (Florianópolis, SC). Normas técnicas para o cultivo de ameixeira em Santa Catarina. 2. ed Florianópolis, 1996. 39 p. (Sistemas de Produção, 22).

EPAGRI (Florianópolis, SC). Normas técnicas para o cultivo de pessegueiro em Santa Catarina. Florianópolis, 1995. 38 p. (Sistemas de Produção, 23).

FORNO, H.; ARRETZ, P.; GONZALES, R. H. Sex pheromone in the detection and evaluation of the oriental fruit moth in Chile. Plant Protection Bulletin, Faridabad, v. 23 , p. $4-11,1975$

GRELMANN, E. O. Exigências térmicas e estimativa do número de gerações de Grapholita molesta (Busk, 1916) (Lepidoptera - Olethreutidae) em Pelotas, RS 1991. 43 f. Dissertação (Mestrado em Fitossanidade) - Universidade Federal de Pelotas, Pelotas, 1991.

GROVER, J. P.; McKEE, D.; YOUNG, S.; GODFRAY, H. C. J.; TURCHIN, P. Periodic dynamics in Daphnia populations: biological interactions and external forcing Ecology, Washington, v. 81, n. 10, p. 2781-2789, Oct. 2000

HALLEY, J. M. Ecology, evolution and 1/f-noise. Trends in Ecology and Evolution, Amsterdam, v. 11, n. 1, p. 33 37, Jan. 1996.

HICKEL, E. R.; DUCROQUET, J. P. H. J. Monitoramento e controle da grafolita ou mariposa oriental no Alto Vale do Rio do Peixe. Agropecuária Catarinense, Florianópolis, v. 11, n. 2, p. 8-11, jun. 1998.

KNELL, R. J. Generation cycles. Trends in Ecology and Evolution, Amsterdam, v. 13, n. 5, p. 186-190, May 1998.

LOGAN, J. A.; ALLEN, J. C. Nonlinear dynamics and chaos in insect populations. Annual Review of Entomology, Palo Alto, v. 37, p. 455-477, 1992.
MIRAMONTES, O.; ROHANI, P. Intrinsically generated colored noise in laboratory insect populations. Proceedings of the Royal Society of London, Series B, London, v. 265, n. 1398, p. 785-792, 1998.

PETCHEY, O. L. Environmental colour affects aspects of single-species population dynamics. Proceedings of the Royal Society of London, Series B, London, v. 267, n. 1445 , p. $747-754,2000$.

PHILLIPS, J. H. H. Monitoring for oriental fruit moth with synthetic sex pheromone. Environmental Entomology, Lanham, v. 2, n. 6, p. 1039-1042, Dec. 1973.

RAI, V.; SCHAFFER, W. M. Chaos in ecology. Chaos, Solitons \& Fractals, Amsterdam, v. 12, n. 2, p. 197-203, Jan. 2001.

RICE, R. E.; BARNETT, W. W.; FLAHERTY, D. L.; BENTLEY, W. J.; JONES, R. A. Monitoring and modeling oriental fruit moth in California. California Agriculture, Davis, v. 36, p. 11-12, Jan./Feb. 1982.

SALLES, L. A. B. Grafolita (Grapholita molesta) bioecologia e controle. Pelotas: Embrapa-CNPFT, 1984. 16 p. (Documentos, 20).

SILVEIRA NETO, S.; PRECETTI, A. A. C. M.; BRAZ, A. J. B. P.; SANTOS, P. E. T. Flutuação populacional de Grapholita molesta (Busk) (Lep., Olethreutidae) em pessegueiro e nectarina com o uso de feromônio sexual sintético. Anais da Sociedade Entomológica do Brasil, Londrina, v. 10, n. 1, p. 43-49, 1981

SKOKOV, V. N.; RESHETNIKOV, A. V.; KOVERDA, V. P.; VINOGRADOV,A. V. Self-organized criticality and $1 /$ f-noise at interacting nonequilibrium phase transitions. Physica A,Amsterdam, v. 293, n. 1/2, p. 1-12, Apr. 2000.

SOLÉ, R. V.; MANRUBIA, S. C.; BENTON, M.; KAUFFMAN, S.; BAK, P. Criticality and scaling in evolutionary ecology. Trends in Ecology and Evolution, Amsterdam, v. 14, n. 4, p. 156-160, Apr. 1999.

TURCHIN, P.; LORIO JUNIOR, P. L.; TAYLOR, A. D.; BILLINGS, R. F. Why do populations of southern pine beetles (Coleoptera: Scolytidae) fluctuate? Environmental Entomology, Lanham, v. 20, n. 2, p. 401-409, Apr. 1991. 